\title{
Altered podocyte structure in GLEPP1 (Ptpro)-deficient mice associated with hypertension and low glomerular filtration rate
}

\author{
Bryan L. Wharram, ${ }^{1}$ Meera Goyal, ${ }^{1}$ Patrick J. Gillespie, ${ }^{2}$ Jocelyn E. Wiggins, ${ }^{1}$ \\ David B. Kershaw, ${ }^{3}$ Lawrence B. Holzman, ${ }^{1}$ Robert C. Dysko, ${ }^{4}$ \\ Thomas L. Saunders, ${ }^{5}$ Linda C. Samuelson, ${ }^{2}$ and Roger C. Wiggins ${ }^{1}$ \\ ${ }^{1}$ Department of Internal Medicine, \\ ${ }^{2}$ Department of Physiology, \\ ${ }^{3}$ Department of Pediatrics, \\ ${ }^{4}$ Unit for Laboratory Animal Medicine, and \\ ${ }^{5}$ Department of Human Genetics, University of Michigan, Ann Arbor, Michigan 48109, USA \\ Address correspondence to: Roger C. Wiggins, University of Michigan Health System, Nephrology Division, \\ 3914 Taubman Center, 1500 E. Medical Center Drive, Ann Arbor, Michigan 48109-0364, USA. \\ Phone: (734) 936-5645; Fax: (734) 936-9621; E-mail: rwiggins@umich.edu.
}

Received for publication May 3, 1999, and accepted in revised form October 3, 2000.

Glomerular epithelial protein 1 (GLEPP1) is a receptor tyrosine phosphatase present on the apical cell surface of the glomerular podocyte. The GLEPP1 gene (Ptpro) was disrupted at an exon coding for the $\mathrm{NH}_{2}$-terminal region by gene targeting in embryonic stem cells. Heterozygote mating produced the expected genotypic ratio of 1:2:1, indicating that the $\mathrm{Ptpro}^{-/}$genotype does not lead to embryonic or neonatal lethality. Kidney and glomerular structure was normal at the gross and light microscopic levels. Scanning and transmission electron microscopy showed that $\mathrm{Ptpro}^{-/}$mice had an amoeboid rather than the typical octopoid structure seen in the wild-type mouse podocyte and that there were blunting and widening of the minor (foot) processes in association with altered distribution of the podocyte intermediate cytoskeletal protein vimentin. Reduced filtration surface area in association with these structural changes was confirmed by finding reduced glomerular nephrin content and reduced glomerular filtration rate in $\mathrm{Ptpro}^{-/-}$mice. There was no detectable increase in the urine albumin excretion of $\mathrm{Ptpro}^{-/-}$mice. After removal of one or more kidneys, $\mathrm{Ptpro}^{-/}$mice had higher blood pressure than did their wild-type littermates. These data support the conclusion that the GLEPP1 (Ptpro) receptor plays a role in regulating the glomerular pressure/filtration rate relationship through an effect on podocyte structure and function.

J. Clin. Invest. 106:1281-1290 (2000).

\section{Introduction}

The glomerulus is an extraordinarily efficient, hydrostatically driven high-volume/high-discrimination filter of blood that is the upstream component of the renal excretory structure called the nephron. Water, ions, and small molecules from blood easily cross the filter, while at the same time the passage of larger blood proteins, particularly the bulk protein of blood (albumin), is minimized. The podocyte (visceral glomerular epithelial cell) is one of the major cell types responsible for maintenance of the structure and function of the glomerular filter.

Podocytes are neuronlike cells with a cell body, major processes, and actin-rich foot processes that abut the glomerular basement membrane (1). These foot processes interdigitate with foot processes of neighboring cells so as to cover the outer filtration surface of glomerular capillaries. Each foot process is attached to its neighbor along its length by an intercellular adherens-type junction modified for filtration (the slit diaphragm) (2). Podocyte foot processes are physically separated from their neighbors in part by an anionic charge effect generated by the major negatively charged sialoprotein of the podocyte, podocalyxin $(3,4)$. Foot processes are attached to the underlying glomerular basement membrane (GBM) by $\alpha 3 \beta 1$ integrins (5). The filtration surface (fenestrated endothelial cells, GBM, and supporting foot processes) is supported, in turn, by the intermediate filament and microtubule containing major and intermediate processes of the podocyte. These podocyte processes serve to counterbalance the pulsatile hydrostatic force of blood pressure driving the filtration process and tending to expand the glomerular capillary lumen (6). The dynamic nature of the filtration structure in which charge plays a critical role is emphasized by the fact that the foot processes can become effaced within 10 minutes after injection of protamine, a positively charged molecule, into the renal artery (7).

Normal function of the filter requires the maintenance of foot-process structure. Injury to the glomerulus is usually associated with leakage of protein across 
the filter into the urine and with disappearance (effacement) of podocyte foot processes either locally or generally (8). Therefore, understanding podocyte biology is essential to understanding how the glomerular filter works and how it becomes disabled in diseases affecting the glomerulus that have loss of protein into the urine as a major clinical feature.

Glomerular epithelial protein 1 (GLEPP1), now also called protein tyrosine phosphatase receptor type $O$ (Ptpro), was identified and cloned in a search for podocyte-specific proteins that might regulate glomerular structure and function (9). GLEPP1 has a single transmembrane domain, a single intracellular phosphatase domain, and a large extracellular domain comprising eight fibronectin type III-like repeats. It is highly conserved between rabbit, rat, mouse, and human, and the gene coding for human GLEPP1 is present on the short arm of chromosome 12 (10). GLEPP1 is expressed on the apical surface of the podocyte early in development and is present on the apical surface of podocyte foot processes in the mature phenotype (11). Thus we have speculated that GLEPP1 may play a role in regulating podocyte structure and function (9-12).

Mutation or disruption of genes coding for several podocyte proteins results in changes in podocyte structure and function and associated filter dysfunction resulting in protein loss into the urine. Mutation of the gene coding for nephrin, a component of the adherenstype junction that forms the slit diaphragm between foot processes, occurs in humans with congenital nephrotic syndrome (Finnish type) and results in failure to form foot processes and in massive protein loss into the urine (13). A similar phenotype is seen in mice with knockout of a nephrin-binding scaffold protein called Cd2ap, which may link nephrin to the actin cytoskeleton (14). Knockouts of $\alpha 3$ integrin, a component of the adhesion molecule complex by which foot processes adhere to the filtration surface (basement membrane), and its potential binding partner in the glomerular basement membrane laminin $\beta 2$, both result in development of effaced foot processes and proteinuria $(5,15)$. Knockout of the podocalyxin gene in the mouse results in failure to form foot processes, proteinuria, and neonatal lethality in the homozygote (16). Genetic analysis of families with proteinuria leading to glomerulosclerosis has identified mutated podocyte proteins involved in cytoskeletal-basement membrane ( $\alpha$-actinin 4$)$ and cytoskeleton-plasma membrane (podocin) interactions $(17,18)$. Thus, disruption of molecules responsible for maintaining the podocyte cytoskeleton and cell shape, particularly those that affect the relationship between foot processes and their neighbors, as well as between foot processes and the underlying basement membrane, result in major changes in structure and function of the glomerular filter. In each case the result is increased leakiness of the glomerular filter manifested by increased loss of protein from blood into the urine.

These considerations led us to expect that if the
GLEPP1 receptor is fulfilling an important role in maintaining foot-process structure, failure to express the GLEPP1 molecule would have a measurable effect on glomerular structure and/or function and would result in increased protein excretion into the urine. We therefore disrupted the GLEPP1 gene (Ptpro) by targeted deletion using homologous recombination in mouse embryonic stem (ES) cells. We obtained GLEPP1-deficient animals from two independent ES cell clones. In both strains of mice we found that GLEPP1 depletion resulted in a modification of podocyte structure such that the normal "octopoid" podocyte structure was simplified to a more "amoeboid" structure and that foot processes were shorter and broader than normal. Instead of finding that the glomerular filtration structure was leaky, however, as described in other examples of podocyte gene disruption, we found that the Ptpro ${ }^{-/}$ mice had reduced glomerular filtration function and a tendency to hypertension.

\section{Methods}

Analysis of the intron/exon structure of the GLEPP1 gene. A genomic library prepared in the Lambda FIX II vector from 129/SvJ mice (catalog no. 946309, Stratagene, La Jolla, California, USA) was screened using a PCR-generated DNA probe corresponding to the $5^{\prime}$ end of the human GLEPP1 cDNA. A 15-kb clone was restrictionmapped and sequenced to define the exon/intron structure of the $5^{\prime}$ region of the GLEPP1 gene (Figure 1).

Construction of the targeting vector. The pPNT targeting vector containing both neomycin phosphotransferase (neo) and herpes simplex virus thymidine kinase genes for positive and negative selection were kindly provided by Richard Mulligan (Children's Hospital, Boston, Massachusetts, USA) (19). A 2.2-kb NotI/SfuI fragment of the $15-\mathrm{kb}$ genomic lambda clone, which included a single NotI site and 20 bp derived from the lambda vector, as well as a portion of the GLEPP1 gene ending at the $S f u$ I site was cloned between the NotI and XhoI sites $5^{\prime}$ of the neo gene by blunt-ended ligation. This resulted in the interruption of putative exon 3 , a frameshift, and introduced a new termination codon. A $5.5-\mathrm{kb}$ XhoI/StuI fragment of the GLEPP1 gene was cloned between the KpnI and EcoRI sites 3' of the neo gene by blunt-ended ligation. DNA sequencing was used to confirm that the appropriate fragments were in the correct orientation. Homologously targeted chromosomes were predicted to have a $7.2-\mathrm{kb}$ deletion of genomic DNA (see Figure 1). The single NotI site $5^{\prime}$ of the 5' GLEPP1 fragment was used to linearize the vector before electroporation.

Targeting of ES cells. Transfection of the linearized vector by electroporation into R1 ES cells (20) grown on a mouse embryonic fibroblast feeder layer was performed as described previously (21) using recombinant leukemia inhibitory factor (ESGRO; Life Technologies Inc., Gaithersburg, Maryland, USA). After selection with either G418 alone or G418 plus ganciclovir, 1,160 
colonies were picked and subcloned in 96-well plates. Colonies were further split into 96-well plates and then frozen pending assay for homologous recombination. DNA prepared from 1,096 clones was restriction-digested with BamHI. Southern blots were then performed for each DNA sample using a $3^{\prime}$ external probe, a $5^{\prime}$ internal probe, and a neo probe as shown in Figure 1. Clones that satisfied criteria for appropriate incorporation at both $3^{\prime}$ and $5^{\prime}$ ends and contained the neo cassette were then thawed, expanded, and assessed for euploidy in metaphase spreads as described previously (21).

Blastocyst injection and mouse-breeding experiments. Two clones with normal chromosome number were microinjected into C57BL/ 6 blastocysts at a concentration of 12-16 ES cells per blastocyst as described (21). The blastocysts were then transferred to the uterus of pseudopregnant female CD-1 mice (Charles River Laboratories, Wilmington, Massachusetts, USA). Pups born to these mice were assessed for chimerism by agouti coat color. Male chimeras were then bred with C57BL/ 6 females to produce offspring heterozygous for the GLEPP1 mutation. Heterozygotes were interbred to produce homozygous offspring. All mice analyzed in this paper were $\mathrm{F}_{2}$ littermates from this cross. Genotype was assessed by PCR to identify $\mathrm{Ptpro}^{+/+}, \mathrm{Ptpro}^{+/-}$, and $\mathrm{Ptpro}^{-/-}$mice. The $3^{\prime}$ primer (5' CAC TGA ATC AAA ATG TCC CAC CCA TGT TTC, denoted by an asterisk in Figure 1) was common to both the wild-type and targeted alleles, and the $5^{\prime}$ primers (wild-type 5' AAA CCT TAA ACT CCT GAT CCT CCT GCC TCC; targeted 5' GCC TTC TAT CGC CTT CTT GAC GAG TTC TTC) were unique to each allele. PCR reactions were performed in a multiplex fashion, and predicted product sizes are 307 and $570 \mathrm{bp}$ for wildtype and targeted alleles, respectively.

Structural analysis. Mice, 4-6 weeks old, from the $\mathrm{Ptpro}^{+/+}, \mathrm{Ptpro}^{+/-}$, and $\mathrm{Ptpro}^{-/}$genotypes were sacrificed by sodium pentobarbital injection. Kidneys were removed to ice, slices were snap-frozen, and cryostat sections were prepared for immunofluorescence using rabbit anti-rat GLEPP1 Ab prepared as described below. For light microscopy $5-\mu \mathrm{m}$ sections were cut from paraffin-embedded tissue and then stained with hematoxylin and eosin. For scanning electron microscopy (SEM), fragments of kidney tissue were fixed in glutaraldehyde/cacodylate buffer and prepared for SEM by sputter coating with gold. Samples were analyzed by an Amray Model 1000B Scanning Electron Microscope (Amray Inc., Bedford, Massachusetts, USA) by examining ten consecutive glomeruli from each sample. Two sets of animals (corresponding to each of the two ES cell clones used to prepare mice) were examined by SEM. The genotype of the sample was known for the first set of three mice examined. To prevent bias in measurements, the genotype of sections under analysis was not revealed to investigators for the second group of three animals examined. Preparation of samples for transmission electron microscopy (TEM) was performed by standard methods using pieces of diced kid- ney fixed in glutaraldehyde/cacodylate buffer. After plastic embedding, $1-\mu \mathrm{m}$ sections were cut and stained with toluidine blue. Selected samples containing glomeruli were thin sectioned and examined by TEM. An investigator blinded to the genotype of the samples performed the quantitative analysis for the TEM photomicrographs.

Preparation of anti-mouse GLEPP1 Ab's and immunofluorescence. The rat GLEPP1 $\mathrm{CDNA}$ was cloned from a rat kidney 5'-STRETCH PLUS cDNA library (CLONTECH Laboratories Inc., Palo Alto, California, USA). A fragment corresponding to fibronectin repeats II through $V$ was amplified by PCR and cloned into the prokaryotic expression vector pGEX-KT (22). The GST fusion protein prepared in Escherichia coli was used to immunize two rabbits. IgG was prepared from rabbit serum by $50 \%$ ammonium sulfate precipitation. This IgG preparation was then passed over a GLEPP1 extracellular domain (ECD) column to which rat GLEPP1 ECD-GST fusion protein had been covalently bound (Amersham Pharmacia Biotech, Piscataway, New Jersey, USA). Bound IgG was eluted at pH 2.5 with glycineHCL buffer. The eluted anti-rat GLEPP1 ECD IgG was then incubated with GST covalently linked to cyanogen bromide-activated beads to remove Ab's specific for GST. The resultant fluoresceinated IgG bound only to glomeruli in a typical pattern previously seen for GLEPP1 in podocytes from human, rat, and rabbit and showed a single band on Western blot at $180 \mathrm{kDa}$ as expected. For GLEPP1 immunofluorescent analysis, cryostat sections of mouse kidney were blocked with $10 \%$ goat and $10 \%$ mouse serum before incubation with immunopurified anti-rat GLEPP1 IgG ( $2 \mu \mathrm{g}$ IgG per section). After washing, bound rabbit IgG was detected with Cy3-conjugated goat anti-rabbit IgG (Jackson ImmunoResearch Laboratories Inc., West Grove, Pennsylvania, USA). Sections were viewed microscopically at $\times 400$ and images recorded with a digital camera. For vimentin immunofluorescence, mouse kidney sections were blocked with $10 \%$ goat serum before incubation with guinea pig anti-bovine vimentin polyclonal serum (catalog no. PAB904; Maine Biotechnology Services Inc., Portland, Maine, USA) followed by fluoresceinated goat anti-guinea pig IgG.

Western and Northern blots. Glomeruli were isolated by iron oxide embolization as described previously (23). Kidneys were perfused with $10-15 \mathrm{ml}$ iron oxide suspension $(5 \mathrm{mg} / \mathrm{ml}$ in normal saline). The average purity of the glomerular preparations was $90 \%$ with no difference between the purity of preparations from $\mathrm{Ptpro}^{+/}$, $\mathrm{Ptpro}^{+/-}$, or $\mathrm{Ptpro}^{-/-}$mice. Glomeruli were suspended at a concentration of 100,000 per milliliter of extraction buffer containing $8 \mathrm{M}$ urea, $1 \%$ Triton X-100, $0.1 \%$ SDS, $2 \mathrm{mM}$ PMSF, $2 \mathrm{mM}$ EDTA, $5 \mathrm{mM}$ n-ethylmalemide in Tris-buffered saline, and sonicated. The glomerular extract supernatant obtained after centrifugation at $14,000 \mathrm{~g}$ for 8 minutes was analyzed by $10 \%$ SDS-PAGE and Western blotting, followed by detection using rabbit anti-rat GLEPP1 immunopurified IgG (see above), 
guinea pig anti-bovine vimentin polyclonal serum (Maine Biotechnology Services Inc.), or preimmune IgG as a negative control. Blots were developed using a horseradish peroxidase-conjugated anti-rabbit or anti-guinea pig enhanced chemiluminescence (ECL) system (Amersham Pharmacia Biotech).

RNA from glomeruli isolated by sieving on ice (average purity 60\%, with no differences between $\mathrm{Ptpro}^{+/}$, $\mathrm{Ptpro}^{+/-}$, and $\mathrm{Ptpro}^{-/-}$mice) was purified using the TRIZOL reagent. RNA was analyzed by Northern blot using a 1-kb human GLEPP1 intracellular domain probe random-primed DNA labeled with $\alpha-{ }^{32} \mathrm{P}$ dCTP (High Prime; Roche Molecular Biochemicals, Indianapolis, Indiana, USA). The blot was stripped and reprobed with human $\beta$-actin cDNA (OriGene Technologies Inc., Rockville, Maryland, USA), similarly labeled to show equal loading. Blots were washed in $0.1 \times$ SSC, $0.1 \%$ SDS before detection by autoradiography.

a

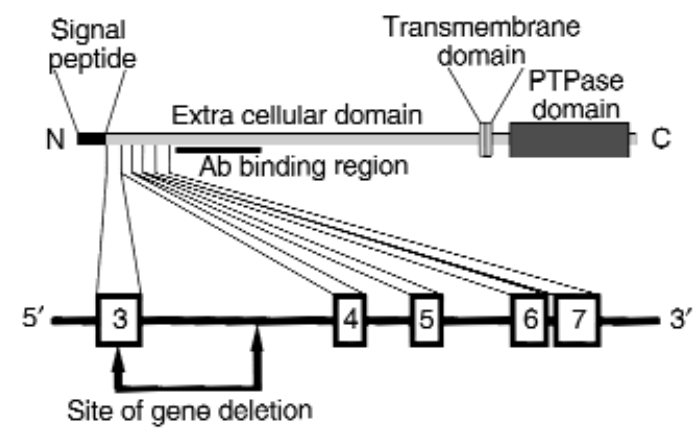

b

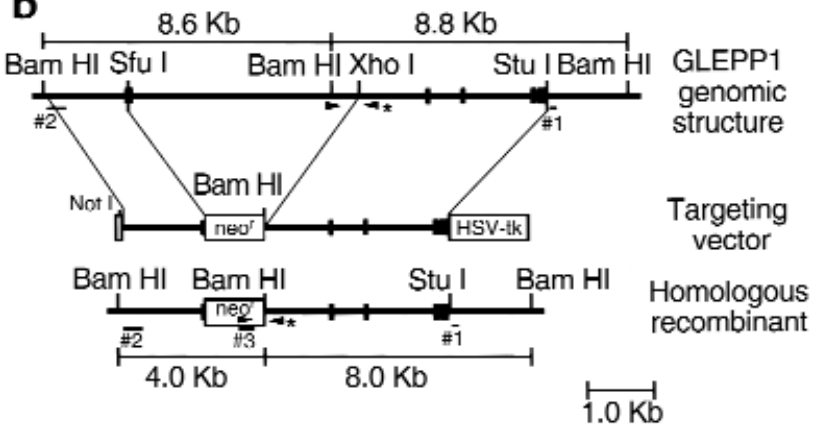

Figure 1

(a) Structure of $5^{\prime}$ end of the mouse GLEPP1 gene showing the exon/intron arrangement in relation to the coding region for the $\mathrm{NH}_{2}$-terminal end of the GLEPP1 protein. Numbered boxes represent exons. Exon 3 is a putative designation pending sequencing of the $5^{\prime}$ region of the gene. The site of gene deletion so as to interrupt an exon, induce a frameshift, and introduce a stop codon is shown. The Ab-binding site, which is $3^{\prime}$ of the deletion, is also shown. (b) Diagrammatic illustrations of the region of the wild-type allele to be targeted (upper), the GLEPP1 gene-targeting vector (middle), and the predicted structure of the targeted allele (lower). Filled boxes correspond to exons 3-7 depicted in a. Sites for restriction enzymes used for constructing the targeting vector and Southern screening of ES cell clones are shown. Size and location of Southern probes are shown, including the $3^{\prime}$ external probe (\#1), the $5^{\prime}$ internal probe $(\# 2)$, and the neo probe (\#3). Arrowheads denote sites for PCR primers used for genotype analysis of mice. The 3' primer, denoted by an asterisk, is common to both the wild-type and targeted alleles.
Proteinuria and creatinine clearance studies. Timed overnight, urine collections from littermates were made by keeping mice in metabolic cages overnight with free access to water, but without food so as to avoid urine contamination. Urine was collected under oil to avoid evaporation. Urine creatinine levels were measured by the alkaline picrate method (catalog no. 555; Sigma, St. Louis, Missouri, USA). After normalizing the urine samples for creatinine levels, they were assayed for total protein using the Bio-Rad microplate protein assay (catalog no. 500-0006; Bio-Rad Laboratories, Hercules, California, USA) and albumin levels by Western blot analysis using rabbit anti-mouse albumin IgG polyclonal antisera (Accurate Chemical \& Scientific Corp., Westbury, New York, USA) and the ECL detection system. A standard curve was created using purified mouse albumin (Sigma). Albumin concentrations were quantified by densitometry. Alternatively, albumin was measured using the microfluoral microalbumin test based on albumin blue 580 (AB580) (catalog no. PR2005; Progen Biotechnik, Heidelberg, Germany) performed in 96-well microplates and measured in a fluorescence microplate reader (excitation $590 \mathrm{~nm}$, emission $620 \mathrm{~nm}$ ). Total protein and albumin concentrations were expressed as amount per milligram of urine creatinine.

Creatinine clearance was calculated for $\mathrm{Ptpro}^{+/+}$and Ptpro $^{-/-}$female littermates $(n=5)$. Timed overnight urine collections were performed using metabolic cages, repeated four times for each individual. Mice were then sacrificed by barbiturate injection and blood was collected by cardiac puncture. Serum and urine creatinine levels were measured by the alkaline picrate method (Sigma). Urine creatinine excretion rates were corrected for time and volume, and the mean was calculated for each individual $(n=4)$. Creatinine clearance was calculated by dividing the mean urine creatinine excretion rates by serum creatinine level, then correcting for body weight (expressed in microliters per minute per gram of body weight).

Blood pressure studies. Systolic blood pressure was measured by tail-cuff plethysmography (catalog no. BP-2000; Visitech Systems, Apex, North Carolina, USA). Systolic blood pressure was measured for ten consecutive cycles on 2 or more days. Mean blood pressures were then calculated for each mouse $(n=20-40)$ and compared between $\mathrm{Ptpro}^{+/+}$and $\mathrm{Ptpro}^{-/-}$female littermates.

Nephrectomy studies. Removal of the right kidney alone or removal of the right kidney and the upper and lower poles of the left kidney (1.4 nephrectomy) was performed as described by Terzi et al. (24). Anesthesia was induced and maintained with isoflurane (Aerrane; Fort Dodge Animal Health, Fort Dodge, Iowa, USA) delivered via a precision vaporizer at $2-4 \%$ concentration. For the 1.4-nephrectomy studies the whole kidney and fragments of kidney removed were weighed so that the approximate proportion of renal tissue removed could be calculated. Renal hemorrhage was controlled by small pledgets of absorbable gelatin 


\section{Figure 2}

(a) Southern blots of BamHI-digested DNA showing lanes with wildtype (WT) and targeted ( $\mathrm{Tg}$ ) alleles hybridized with probes for the $3^{\prime}$ (\#1) and $5^{\prime}(\# 2)$ ends of the targeted region as defined in Figure 1. (b) PCR analyses of tail DNA from $\mathrm{Ptpro}^{+/+}, \mathrm{Ptpro}^{+/-}$, and $\mathrm{Ptpro}^{-/-}$ mice, showing the assay system used to identify genotypes.

(Gelfoam; Pharmacia \& Upjohn Co., Kalamazoo, Michigan, USA). Abdominal musculature was closed with 4-0 polydioxanone suture (PDSII; Ethicon Inc., Somerville, New Jersey, USA), and the skin incision was closed with wound clips.

Commercially acquired Ab's. Ab's used include $\alpha 3$-integrin (Chemicon International, Temecula, California, USA), $\alpha$-actinin, focal adhesion kinase, FITC-labeled phalloidin (Sigma), tubulin (Cortex Biochem Inc., San Leandro, California, USA), ZO-1 (Zymed Laboratories Inc., South San Francisco, California, USA), and vimentin (Maine Biotechnology Services Inc.).

Statistics. Calculations for the genotypic analysis were performed using the $\chi$-squared test. Statistical significance for the data in Table 1 was assessed using the Student's $t$ test (two-sample assuming equal variance).

\section{Results}

Homologous recombination in ES cells. Figure 1a shows the targeting strategy whereby the putative exon 3 coding

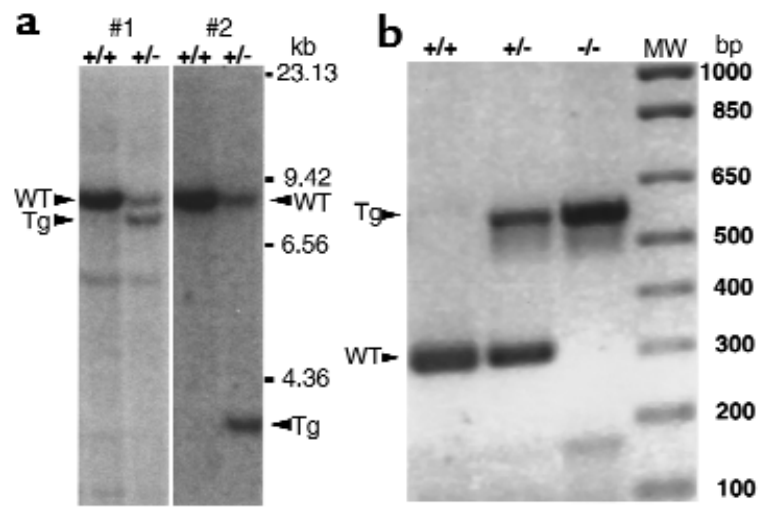

for the $\mathrm{NH}_{2}$-terminal region of the mature GLEPP1 protein would be interrupted by homologous recombination. A truncated GLEPP1 protein consisting of a signal peptide and the $\mathrm{NH}_{2}$-terminal 41 amino acids of the mature protein is the expected translation product. The binding site of the polyclonal $\mathrm{Ab}$ recognizing mouse GLEPP1 is shown to illustrate that homologous recombination would not delete the DNA sequence encoding the Ab-binding region.

Figure $1 \mathrm{~b}$ shows the design of the targeting construct. Subclones of ES cells grown in the presence of selective agents (G418 alone or with ganciclovir) were assessed by Southern blotting of DNA digested with BamHI and probed with a $3^{\prime}$ external probe (probe 1), a $5^{\prime}$ internal

Table 1

Structural and functional measurements in Ptpro $^{+/+}$and Ptpro ${ }^{-/}$mice

\begin{tabular}{|c|c|c|c|c|c|c|}
\hline Parameter measured & Sex & $n$ & \# kidneys & Ptpro $^{+/+}$ & Ptpro $^{-1-}$ & $P$ value \\
\hline $\begin{array}{l}\text { Structural measurements } \\
\text { Mean foot-process width }(\mathrm{nm}) \\
\text { Mean slit-diaphragm width }(\mathrm{nm}) \\
\text { Glomerular nephrin }(\% \text { control) } \\
\text { Glomerular podocalyxin (\% control) }\end{array}$ & $\begin{array}{l}- \\
- \\
-\end{array}$ & $\begin{array}{c}200 \\
40 \\
4 \\
4\end{array}$ & $\begin{array}{l}2 \\
2 \\
2 \\
2\end{array}$ & $\begin{array}{r}322 \pm 19 \\
38.5 \pm 1.1 \\
100 \pm 0.0 \\
100 \pm 0.0\end{array}$ & $\begin{array}{l}425 \pm 19 \\
38.6 \pm 0.9 \\
74.5 \pm 6.8 \\
96.5 \pm 22.8\end{array}$ & $\begin{array}{l}<0.01 \\
\text { NS } \\
<0.01 \\
\text { NS }\end{array}$ \\
\hline $\begin{array}{l}\text { Functional measurements } \\
\text { Serum creatinine }(\mathrm{mg} \%) \\
\text { Serum creatinine }(\mathrm{mg} \%) \\
\text { Creatinine clearance }(\mu \mathrm{l} / \mathrm{min} / \mathrm{g} \text { body weight })\end{array}$ & $\begin{array}{l}M \\
F \\
F\end{array}$ & $\begin{array}{l}5 \\
5 \\
5\end{array}$ & $\begin{array}{l}2 \\
1 \\
1\end{array}$ & $\begin{array}{l}0.36 \pm 0.05 \\
0.37 \pm 0.04 \\
1.76 \pm 0.45\end{array}$ & $\begin{array}{l}0.35 \pm 0.03 \\
0.51 \pm 0.04 \\
0.93 \pm 0.06\end{array}$ & $\begin{array}{l}\text { NS } \\
0.02 \\
0.05\end{array}$ \\
\hline $\begin{array}{l}\text { Urine protein/creatinine ratio }(\mathrm{mg} / \mathrm{mg}) \\
\text { Urine protein/creatinine ratio }(\mathrm{mg} / \mathrm{mg}) \\
\text { Urine protein/creatinine ratio }(\mathrm{mg} / \mathrm{mg}) \\
\text { Urine protein/creatinine ratio }(\mathrm{mg} / \mathrm{mg}) \\
\text { Urine protein/creatinine ratio }(\mathrm{mg} / \mathrm{mg})\end{array}$ & $\begin{array}{l}M \\
F \\
F \\
F\end{array}$ & $\begin{array}{c}8 \\
10 \\
5 \\
5 \\
3\end{array}$ & $\begin{array}{l}2 \\
2 \\
1 \\
0.6 \\
0.6\end{array}$ & $\begin{array}{l}8.4 \pm 1.2 \\
3.2 \pm 0.5 \\
2.7 \pm 0.3 \\
2.9 \pm 0.6 \\
3.1 \pm 0.6\end{array}$ & $\begin{array}{l}7.4 \pm 1.0 \\
4.3 \pm 1.4 \\
2.9 \pm 0.3 \\
3.7 \pm 1.2 \\
3.9 \pm 1.6\end{array}$ & $\begin{array}{l}\text { NS } \\
\text { NS } \\
\text { NS } \\
\text { NS } \\
\text { NS }\end{array}$ \\
\hline $\begin{array}{l}\text { Urine albumin/creatinine ratio }(\mu \mathrm{g} / \mathrm{mg})^{\mathrm{A}} \\
\text { Urine albumin/creatinine ratio }(\mu \mathrm{g} / \mathrm{mg})^{\mathrm{A}} \\
\text { Urine albumin/creatinine ratio }(\mu \mathrm{g} / \mathrm{mg})^{\mathrm{A}} \\
\text { Urine albumin/creatinine ratio }(\mu \mathrm{g} / \mathrm{mg})^{\mathrm{A}} \\
\text { Urine albumin:/creatinine ratio }(\mu \mathrm{g} / \mathrm{mg})^{\mathrm{B}} \\
\text { Urine albumin/creatinine ratio }(\mu \mathrm{g} / \mathrm{mg})^{\mathrm{B}}\end{array}$ & $\begin{array}{l}M \\
F \\
F \\
F \\
M \\
F\end{array}$ & $\begin{array}{c}13 \\
11 \\
5 \\
6 \\
10 \\
5\end{array}$ & $\begin{array}{c}2 \\
2 \\
1 \\
0.6 \\
2 \\
2\end{array}$ & $\begin{aligned} 206 & \pm 40 \\
59 & \pm 10 \\
34 & \pm 7 \\
130 & \pm 72 \\
362 & \pm 79 \\
108 & \pm 53\end{aligned}$ & $\begin{array}{r}200 \pm 27 \\
88 \pm 18 \\
61 \pm 14 \\
66 \pm 16 \\
370 \pm 69 \\
100 \pm 21\end{array}$ & $\begin{array}{l}\text { NS } \\
\text { NS } \\
\text { NS } \\
\text { NS } \\
\text { NS } \\
\text { NS }\end{array}$ \\
\hline $\begin{array}{l}\text { Blood-pressure measurements } \\
\text { Systolic blood pressure }(\mathrm{mmHg}) \\
\text { Systolic blood pressure }(\mathrm{mmHg}) \\
\text { Systolic blood pressure }(\mathrm{mmHg}) \\
\text { Systolic blood pressure }(\mathrm{mmHg})\end{array}$ & $\begin{array}{l}F \\
F \\
F \\
F\end{array}$ & $\begin{array}{c}12 \\
5 \\
5 \\
3\end{array}$ & $\begin{array}{c}2 \\
1 \\
0.6 \\
0.6\end{array}$ & $\begin{array}{r}100.3 \pm 3.4 \\
99.7 \pm 1.7 \\
93.8 \pm 2.9 \\
95.4 \pm 3.6\end{array}$ & $\begin{array}{l}102.2 \pm 2.5 \\
106.9 \pm 2.7 \\
106.9 \pm 2.3 \\
104.8 \pm 0.9\end{array}$ & $\begin{array}{c}\text { NS } \\
0.02 \\
<0.01 \\
0.06\end{array}$ \\
\hline
\end{tabular}

The table shows data for male and female mice with intact kidneys, uninephrectomy, or 1.4 nephrectomy. Structural measurements: The mean width of foot processes was calculated from TEM measurements. For filtration slit width the space between well-defined foot processes on $\times 18,000$ TEM photomicrographs was measured. Nephrin and podocalyxin measurements were made by densitometric analysis of Western blots developed with specific Ab's and using purified glomerular extracts from tpro $^{+/+}$and Ptpro ${ }^{-/-}$mice. Functional measurements: The urine protein/creatinine ratio and urine albu$\mathrm{min} / \mathrm{creatinine} \mathrm{ratios} \mathrm{were} \mathrm{measured} \mathrm{on} \mathrm{urine} \mathrm{collected} \mathrm{in} \mathrm{metabolic} \mathrm{cages.} \mathrm{Two} \mathrm{different} \mathrm{methods} \mathrm{were} \mathrm{used} \mathrm{for} \mathrm{albumin} \mathrm{measurement:}{ }^{\mathrm{A}} \mathrm{AB} 580$ fluorescence assay; BWestern blot. Creatinine clearance was measured using the mean of four 18- to 24-hour timed collections for each animal. Blood pressure measurements: Systolic blood pressure was measured using the tail-cuff method. Individual values were calculated as the mean of at least 20 measurements for each animal. Data shown are the mean \pm SEM for Ptpro ${ }^{+/+}$and Ptpro ${ }^{-/-}$female littermates. NS, not significant. 




Figure 3

(a) Western blot of proteins extracted from glomeruli isolated from $\mathrm{Ptpro}^{-/-}, \mathrm{Ptpro}^{+/-}$, and Ptpro ${ }^{+/+}$mice. The GLEPP1 band is at $180 \mathrm{kDa}$. Bands at lower molecular weights are nonspecific and were seen at the same intensities on a control blot developed with preimmune Ab's (not shown). Numbers below lanes correspond to micrograms of glomerular Triton protein extract loaded onto each lane. Ptpro ${ }^{+/+}$ extract was loaded at twofold serial dilutions from the right side of the gel and could be detected at the 32-fold dilution. GLEPP1 was not detectable in tpro $^{-/-}$extracts. GLEPP1 was present in Ptpro ${ }^{+/}$ extracts at a level approximately one-half that of extracts from $\mathrm{Ptpro}^{+/+}$mice. (b) Northern blot of RNA prepared from glomeruli isolated from $\mathrm{Ptpro}^{+/+}, \mathrm{Ptpro}^{+/-}$, and $\mathrm{Ptpro}^{-/-}$mice. Left panel: probed with $1 \mathrm{~kb}$ human GLEPP1 intracellular domain, random-primed DNA labeled with $\alpha{ }^{32} \mathrm{P}$ dCTP. Right panel: probed with human $\beta$ actin probe to show equal loading of RNA onto the lanes. No detectable hybridization was seen in the Ptpro-/- RNA sample.

probe (probe 2), and a probe from the neo gene (probe $3)$. The predicted restriction patterns based on DNAsequence information for probe 1 were 8.8 and $8.0 \mathrm{~kb}$ for wild-type and targeted alleles, respectively, and for probe 2 were 8.6 and $4.0 \mathrm{~kb}$ for wild-type and targeted alleles, respectively. Figure 2a shows data from a Southern blot for a successfully targeted clone. Two successfully targeted euploid clones (P5G9 and P4B6) were used to produce ES cell mouse chimeras.

Genotypic and gross phenotypic analysis. Chimeric males were mated with C57BL/6 females to transmit the targeted mutation. Heterozygotes were then interbred, and the progeny were genotyped by PCR to identify $\mathrm{Ptpro}^{+/+}, \mathrm{Ptpro}^{+/-}$, and $\mathrm{Ptpro}^{-/-}$mice (Figure 2b). The proportion of $\mathrm{Ptpro}^{+/+}, \mathrm{Ptpro}^{+/-}$, and $\mathrm{Ptpro}^{-/-}$mice derived from clone P5G9 were $29.5 \%, 47.3 \%$, and $23.2 \%$, respectively $(n=220)$, and that of mice derived from clone P4B6 were $24.6 \%, 56.9 \%$, and $18.5 \%$, respectively $(n=65)$. These values were not statistically different from the expected 1:2:1 ratio. No differences were observed in gross appearance, in body weight, or at autopsy between the $\mathrm{Ptpro}^{+/+}, \mathrm{Ptpro}^{+/-}$, and $\mathrm{Ptpro}^{-/-}$mice. Furthermore, matings between $\mathrm{Ptpro}^{-/-}$ mice produced apparently normal offspring, indicating that the Ptpro deletion did not affect fertility, ability to carry a fetus to term, or ability to nourish offspring to weaning at 3 weeks of age.

Quantitation of glomerular GLEPP1 depletion in Ptpro-/mice. To assess the degree of GLEPP1 depletion in the $\mathrm{Ptpro}^{-/-}$mice, glomeruli were isolated from the kidneys of $\mathrm{Ptpro}^{+/}$, Ptpro ${ }^{+/-}$, and $\mathrm{Ptpro}^{-/}$mice, and protein extracts were analyzed by Western blot as shown in Figure $3 \mathrm{a}$. Twofold serial dilutions of extracted proteins from the $\mathrm{Ptpro}^{+/+}$mouse were made to determine the sensitivity of the assay. The band at $180 \mathrm{kDa}$ corresponding to GLEPP1 was detectable at a 1:32 dilution of the wild-type glomerular extract. No detectable band was present in $\mathrm{Ptpro}^{-/-}$extracts. The band in the $\mathrm{Ptpro}^{+/-}$ extract was approximately equal to a dilution of onehalf of the wild-type, indicating that GLEPP1 levels in $\mathrm{Ptpro}^{+/-}$mice were approximately 50\% of GLEPP 1 levels in $\mathrm{Ptpro}^{+/+}$mice. These results were the same for mice obtained from both ES cell clones (data not shown). In a separate experiment (data not shown), threefold serial dilutions of the $\mathrm{Ptpro}^{+/+}$glomerular extract showed a detectable signal at a 1:81 dilution, while no signal was detectable in glomerular extracts from $\mathrm{Ptpro}^{-/-}$mice. We conclude that the GLEPP1 protein in tpro $^{-/-}$mice is present at less than $1.2 \%$ that found in wild-type mice.

To assess whether alternative splicing had occurred, we performed Northern blots with RNA prepared from glomeruli and a cDNA probe from the GLEPP1 phosphatase domain. No detectable hybridization was seen in the $\mathrm{Ptpro}^{-/}$mouse glomerular RNA (Figure 3b). Stripping the blot and reprobing for $\beta$-actin showed similar loading of all samples. These results indicate that a major alternately spliced version of GLEPP1 (that would be expected to hybridize with the downstream GLEPP1 phosphatase-region probe) was not detectably transcribed in the glomeruli of $\mathrm{Ptpro}^{-/}$mice.

Immunofluorescence analysis of $\mathrm{Ptpro}^{-/-}$kidneys. Cryostat kidney sections from wild-type $\left(\right.$ Ptpro $\left.^{+/+}\right)$, heterozygous $\left(\mathrm{Ptpro}^{+/-}\right)$, and homozygous $\left(\mathrm{Ptpro}^{-/-}\right)$mice incubated with GLEPP1 ECD Ab are shown in Figure 4. The fluorescent signal was confined to the glomerulus as reported previously for the rabbit and human $(9,10)$. Bright fluorescence was seen in the $\mathrm{Ptpro}^{+/+}$mice, less intense fluorescence was seen in the $\mathrm{Ptpro}^{+/-}$mice, and markedly reduced but still positive
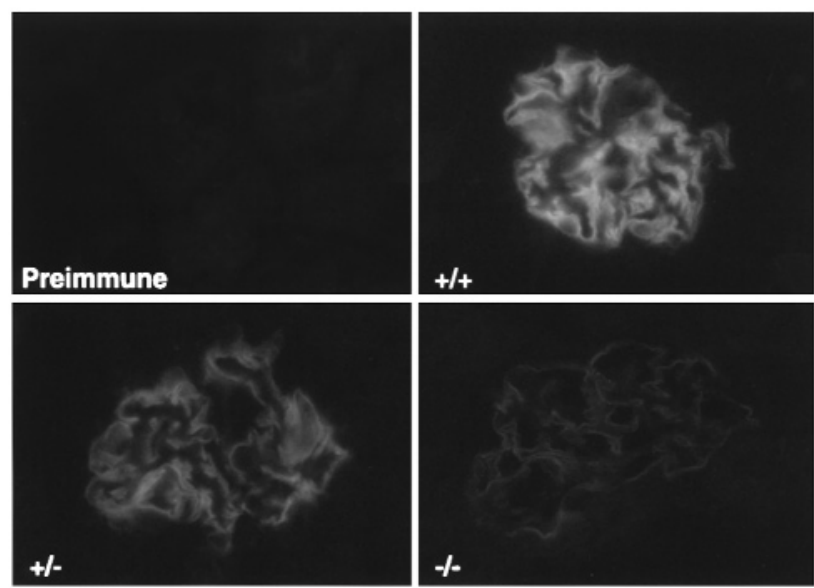

\section{Figure 4}

Indirect immunofluorescence of mouse renal cortex cryostat sections developed using rabbit anti-rat GLEPP1 ECD IgG ( tpro $^{+/+}$, upper right; Ptpro ${ }^{+/-}$, lower left; and $P t_{p r o}^{-/-}$, lower right). Preimmune IgG tested on a $\mathrm{tpro}^{+/+}$renal cortical section is shown at upper left. The photomicrographs are developed to the same intensity of signal. $\times 400$. 
Figure 5

Scanning electron micrographs of glomeruli made at different magnifications, with the wild-type $\left(\mathrm{Ptpro}^{+/+}\right)$ mice shown in the left three panels and $\mathrm{Ptpro}^{-/-}$mice shown in the right three panels. The major processes seen in $\mathrm{Ptpro}^{-/-}$mice were broader, wider, and less distinct than the narrower, more well-defined major processes seen in the wild-type mice. The overall structure of the Ptpro-/- podocytes is more amoeba-like compared with the octopus-like structure of the wildtype $(\times 3,000$, top panels). At high power the foot processes of the $\mathrm{Ptpro}^{+/+}$mice can be seen to be regular and fingerlike in appearance in contrast to the broader, shorter (more toelike) foot processes found in the Ptpro $^{-/}$mice $(\times 10,000$, bottom panels $)$.

fluorescence was seen in the $\mathrm{Ptpro}^{-/-}$mice. This faint fluorescence in the $\mathrm{Ptpro}^{-/-}$ mice could be absorbed out by rat GLEPP1-GST fusion protein, suggesting that $\mathrm{Ab}$ binding was specific. Furthermore, when anti-GLEPP1 Ab's were used to immunoprecipitate GLEPP1 from glomerular extracts made up from four $\mathrm{Ptpro}^{-/-}$mouse kidneys and analyzed by Western blot, a faint band at much greater than $500 \mathrm{kDa}$ (top of the stacking gel) was detected (data not shown). This result suggests that a polypeptide recognized by the anti-GLEPP $1 \mathrm{Ab}$ is made in the podocytes of $\mathrm{Ptpro}^{-/-}$mice, but it is markedly reduced in amount $(<1.2 \%)$ and is part of a polypeptide or glycopeptide, which is several times larger than the native GLEPP1 glycosylated protein.

Renal structure at the light microscopic level. Glomerular histology from 4- to 6-week-old mice was examined. Examination of 5- $\mu \mathrm{m}$ kidney sections stained with hematoxylin and eosin, as well as photomicrographs of plastic-embedded $1-\mu \mathrm{m}$ sections stained with toluidine blue, showed no significant differences between $\mathrm{Ptpro}^{+/+}, \mathrm{Ptpro}^{+/-}$, and $\mathrm{Ptpro}^{-/-}$mice (data not shown).

Ultrastructure analysis of podocytes. SEM showed an obvious difference between $\mathrm{Ptpro}^{+/+}$and $\mathrm{Ptpro}^{-/-}$mice in general podocyte structure (Figure 5). Major processes were blunted and widened in the Ptpro- ${ }^{-/}$mice so that the cells appeared amoeboid rather than octopoid $(\times 3,000$, top panels). Foot processes appeared shortened and thickened (toelike) in $\mathrm{Ptpro}^{-/-}$mice in contrast to the usual thin, even fingerlike processes seen in wild-type mice $(\times 10,000$, bottom panels). No significant difference was apparent between $\mathrm{Ptpro}^{+/+}$and $\mathrm{Ptpro}^{+/-}$mice (data not shown). The same result was obtained from SEM analysis of mice derived from each of the two ES cell clones.

Transmission electron micrographs of kidneys from $\mathrm{Ptpro}^{+/+}, \mathrm{Ptpro}^{+-}$, and $\mathrm{Ptpro}^{-/-}$mice (strain P5G9) are shown in Figure 6. No difference was seen between $\mathrm{Ptpro}^{+/+}$and $\mathrm{Ptpro}^{+/-}$mice. However, foot processes appeared to be widened in $\mathrm{Ptpro}^{-/-}$mice. To assess this quantitatively, the number of intercellular junctions
$+/+$
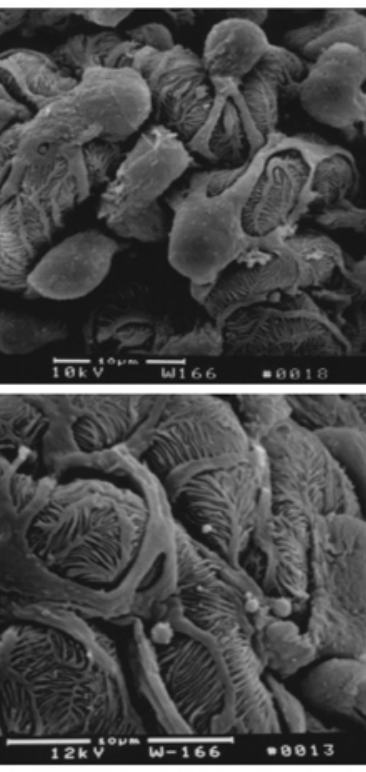

$12 \mathrm{k}$

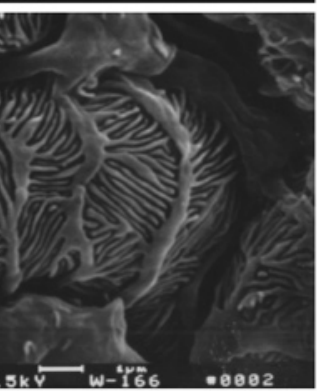

$-1$
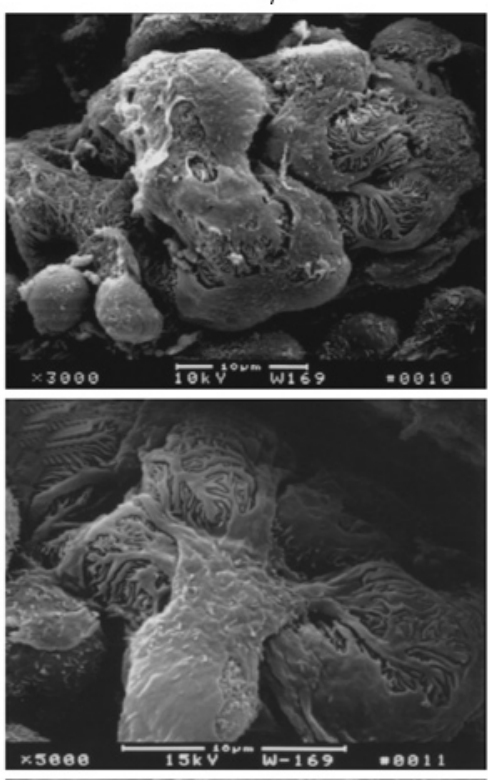

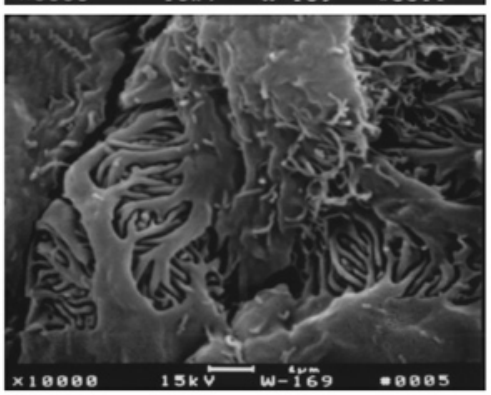

per 5- $\mu \mathrm{m}$ length of GBM was counted in regions adjacent to the urinary space (Table 1 ). There was a statistically significant reduction in number of intercellular junctions per unit of GBM length in the $\mathrm{Ptpro}^{-/-}$mice compared with the $\mathrm{Ptpro}^{+/+}$and $\mathrm{Ptpro}^{+/-}$mice $(P<0.01)$. Table 1 also shows that the gap between foot processes was not different between $\mathrm{Ptpro}^{-/-}$and wild-type mice, indicating that the difference noted above was due to foot-process width rather than the gap between foot processes. This was confirmed in a second set of measurements made on photomicrographs from kidneys from mice derived from clone P4B6. The width of consecutive processes abutting the GBM along the total length of glomerular capillary loops facing the urinary space was measured directly using a micrometer gauge on $\times 18,000$ photomicrographs. The mean width of these processes for the $\mathrm{Ptpro}^{-/-}$animals was significantly wider than for the $\mathrm{Ptpro}^{+/-}$or $\mathrm{Ptpro}^{+/+}$animals (Figure 7). This result supports the SEM findings described above, where the shorter and broader toelike processes would be expected to be transected over a wider range of widths than the longer, more narrow, fingerlike processes in the wild-type mice.

Analysis of vimentin in glomeruli of $\mathrm{Ptpro}^{-/-}$and $\mathrm{Ptpro}^{+/+}$ mice. Vimentin is a major component of intermediate filaments present in the major processes of podocytes (1). We therefore performed immunofluorescent analysis to assess the distribution of vimentin in $\mathrm{Ptpro}^{-/-}$and 

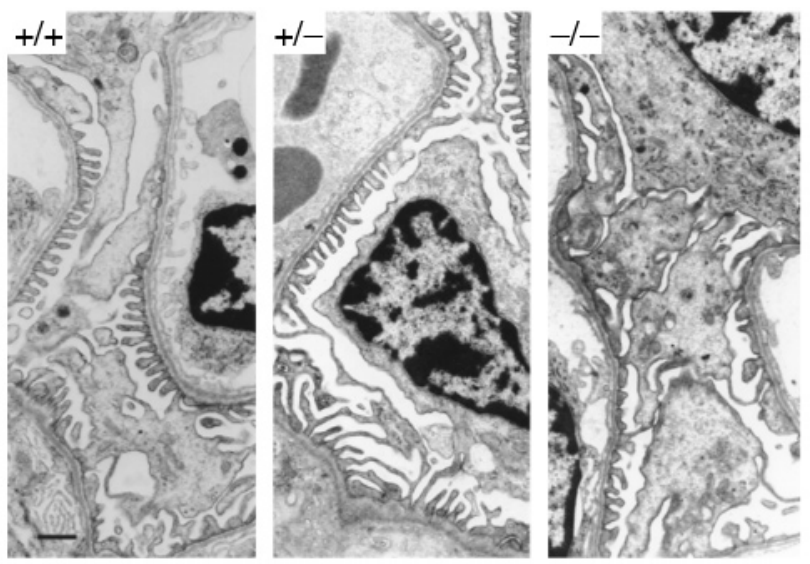

Figure 6

Transmission electron micrographs showing glomerular capillary loops from $\mathrm{Ptpro}^{+/+}, \mathrm{Ptpro}^{+/-}$, and $\mathrm{Ptpro}^{-/-}$mice, revealing broader foot process structure in the tpro $^{-1-}$ mice. Bar, $1 \mu \mathrm{m}$.

wild-type mice (Figure 8a). Vimentin appeared to have an altered distribution in $\mathrm{Ptpro}^{-/-}$mice. In addition, there was a more intense immunofluorescent signal in $\mathrm{Ptpro}^{-/-}$glomeruli. To examine this more quantitatively we performed Western blots using glomerular extracts from $\mathrm{Ptpro}^{-/-}$and wild-type mice. Glomeruli were sequentially extracted (a) with Triton alone, (b) with sonication in the Triton buffer to solubilize cytoskeletal elements, and (c) after SDS extraction of the remaining pellet. As shown in Figure 8b, vimentin was increased in $\mathrm{Ptpro}^{-/-}$glomeruli. We conclude that there is an increased amount of vimentin in $\mathrm{Ptpro}^{-/}$ glomeruli, as well as a redistribution of the vimentin.

Indirect immunofluorescence on methanol-fixed cryostat sections was used to assess distribution of the following proteins: $\alpha 3$-integrin, $\alpha$-actinin, cytokeratin, focal adhesion kinase, phalloidin FITC-labeled, tubulin, and ZO-1. No major differences were apparent between $\mathrm{Ptpro}^{+/+}$and $\mathrm{Ptpro}^{-/-}$mice (data not shown).

Quantitation of nephrin and podocalyxin in glomeruli. The average width of the foot process of the $\mathrm{Ptpro}^{-/-}$mice was $425 \pm 19 \mathrm{~nm}$ compared with a value of $322 \pm 19$ $\mathrm{nm}$ for the $\mathrm{Ptpro}^{+/+}$mice (Table 1). The slit diaphragm was the same width in both groups of animals (approximately $39 \mathrm{~nm}$ ). Thus, the calculated proportion of the GBM length that is available for passage of filtrate between foot processes for $\mathrm{Ptpro}^{+/+}$mice is $10.8 \%$ compared with $8.4 \%$ in $\mathrm{Ptpro}^{-/-}$mice. Therefore, the podocyte intercellular space of $\mathrm{Ptpro}^{-/}$mice is approximately $78 \%$ that of $\mathrm{Ptpro}^{+/+}$mice. Since nephrin is an important component of the intercellular junction, one might expect that there would be less nephrin in glomeruli of $\mathrm{Ptpro}^{-/-}$mice than their wildtype littermates. Glomeruli were purified from $\mathrm{Ptpro}^{+/+}$ and $\mathrm{Ptpro}^{-/-}$mice, extracted, and analyzed for nephrin by Western blot. Podocalyxin, a glycoprotein present on both the podocyte and glomerular endothelial cell, was used as a control. Experiments were done isolating glomeruli from a matched pair of $\mathrm{Ptpro}^{+/+}$and
$\mathrm{Ptpro}^{-/-}$mice, and in each case the data were normalized to $100 \%$ for the $\mathrm{Ptpro}^{+++}$mouse of the pair. The results for nephrin and podocalyxin quantitation as assessed by densitometry of the blot are shown in Table 1. Glomeruli of $\mathrm{Ptpro}^{-/-}$mice contained approximately $25 \%$ less nephrin than the $\mathrm{Ptpro}^{+/+}$controls. In contrast, the podocalyxin content was not significantly different between $\mathrm{Ptpro}^{+/+}$and $\mathrm{Ptpro}^{-/-}$mice. This result is consistent with the conclusion that $\mathrm{Ptpro}^{-/-}$ mice have less foot processes per unit area of GBM and therefore less filtration area, as would be expected from the structural studies.

Measurement of glomerular filtration rate. As reported above, the filtration surface of $\mathrm{Ptpro}^{-/-}$mice is about 75\% that of $\mathrm{Ptpro}^{+/+}$mice. This difference might be reflected in measurable differences in glomerular filtration rate (GFR). Initial serum creatinine measurements in blood from intact mice showed no significant difference between $\mathrm{Ptpro}^{+/+}$and $\mathrm{Ptpro}^{-/-}$mice (Table 1). When mice were uninephrectomized to reduce renal reserve, however, the $\mathrm{Ptpro}^{-/-}$mice were found to have $25-50 \%$ lower GFR than their wild-type littermates as assessed by either creatinine clearance per gram of body weight or serum creatinine concentration.

Measurement of protein and albumin excretion. Groups of male and female mice were assessed for excretion of protein and albumin in the urine (Table 1). Albumin excretion was quantified using an AB580 fluorescence assay as well as by Western blotting to visualize intact albumin molecules. There was no difference in either total protein or albumin excretion between $\mathrm{Ptpro}^{+/+}$and $\mathrm{Ptpro}^{-/-}$mice. After uninephrectomy to reduce renal reserve, we were still unable to detect a difference in protein or albumin excretion between $\mathrm{Ptpro}^{+/+}$and $\mathrm{Ptpro}^{-/-}$mice. To assess the effect of removal of more than one kidney, a group of mice was subjected to 1.4 nephrectomy. Six pairs of mice were nephrectomized in a randomized fashion without the surgeon being aware of the genotype. The mean amount of kidney removed from each mouse was meas-

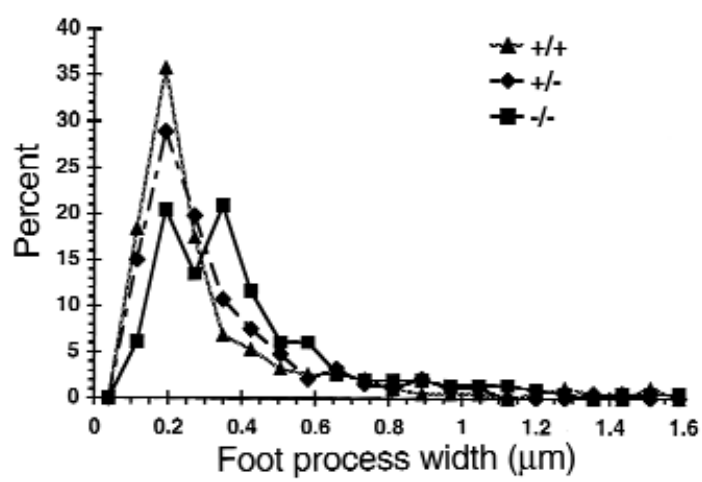

Figure 7

Graph of the distribution of foot-process width measured on TEM in regions of open glomerular capillaries from $\mathrm{Ptpro}^{+/+}$(triangle), $\mathrm{Ptpro}^{+/-}$(diamond), and Ptpro-/- (square) animals. The Ptpro ${ }^{+/+}$and $\mathrm{Ptpro}^{+/-}$mice have narrow foot processes predominantly in the 0.2 $\mu \mathrm{m}$ range, whereas the $P$ tpro $^{-/-}$mice have a significantly broader range of foot-process width. 


\section{Figure 8}

(a) Immunofluorescence photomicrographs developed with anti-vimentin $\mathrm{Ab}^{\prime}$ 's in $\mathrm{Ptpro}^{+/+}$(left) and $\mathrm{Ptpro}^{-/-}$(right) glomeruli showing the different distribution of vimentin and apparent increase in amount in the tpro $^{-/-}$mice. (b) Western blot of glomerular extracts from $\mathrm{Ptpro}^{-/-}$and $\mathrm{Ptpro}^{+/+}$mice made using differential extraction by Triton alone (right two lanes), Triton plus sonication to solubilize cytoskeletal proteins (middle two lanes), and the SDS-extracted pellet (left two lanes). The signal for vimentin (top) suggests that vimentin is present in increased amount in the $\mathrm{Ptpro}^{-/-}$mice. Approximately equal protein loading of glomerular extracts is shown by Coomassie blue-stained gels (bottom).
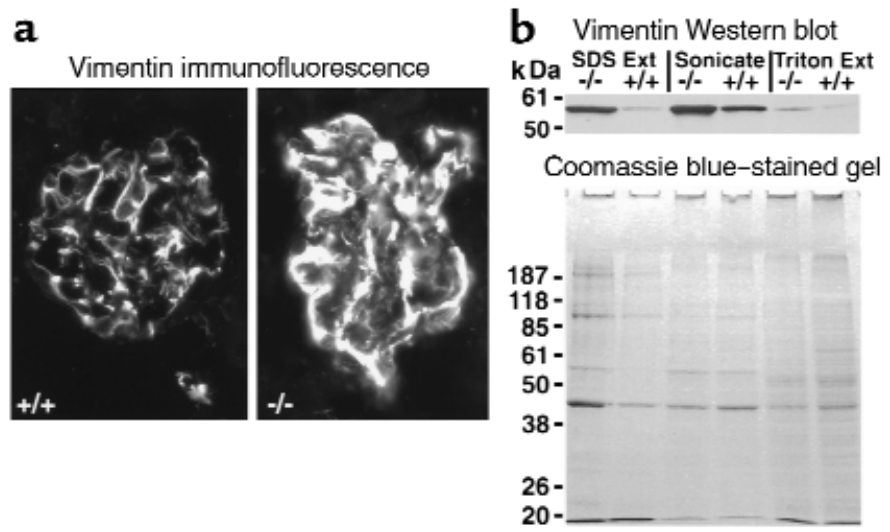

ured. The amount of kidney removed from the Ptpro-/mice was significantly greater than that removed from the $\mathrm{Ptpro}^{+/+}$mice $(1.44 \pm 0.03$ vs. $1.34 \pm 0.02, P<0.05)$. Thus, for further analysis as shown in Table 1, we also present data for three matched pairs of 1.4-nephrectomized mice that had $1.38 \pm 0.01$ and $1.42 \pm 0.04 \mathrm{kid}-$ neys removed for the $\mathrm{Ptpro}^{+/+}$and $\mathrm{Ptpro}^{-/-}$mice, respectively. The 1.4-nephrectomized Ptpro-/- mice did not excrete more protein or albumin in their urine compared with the wild-type mice. We conclude from these studies that the $\mathrm{Ptpro}^{-/-}$mice do not have increased urinary protein or albumin excretion, even under conditions of reduced renal reserve.

Predisposition to bypertension in Ptpro ${ }^{-/-}$mice. Blood pressure was measured in non-nephrectomized, uninephrectomized, and 1.4-nephrectomized mice using the tail-cuff method. As shown in Table 1, the non-nephrectomized Ptpro $^{-/-}$mice showed no significant difference in blood pressure compared with wildtype mice, when kept on a standard chow diet. However, when mice were uninephrectomized or had 1.4 kidneys removed, the Ptpro-/- groups had significantly higher blood pressure than did the wild-type controls. Thus Ptpro $^{-/}$mice have a predisposition to hypertension, which becomes easily detectable when renal mass is reduced.

\section{Discussion}

The experiments described show that disruption of the GLEPP1 gene resulted in a change in podocyte structure. The Ptpro-/- podocytes were more amoeboid in shape, while wild-type podocytes had a well-defined octopoid appearance. In addition, $\mathrm{Ptpro}^{-/-}$mouse foot processes were shorter and broader than those of the normal fingerlike structure found in wild-type mice. These alterations in podocyte structure were accompanied by a change in the distribution of vimentin, a major intermediate filament of the podocyte. Vimentin polymerization is regulated through phosphorylation of serine/threonine, as well as tyrosine residues, and tyrosine kinases can modify vimentin organization in cells $(25,26)$. Therefore, potential mechanisms to explain the observed effect might involve regulation of the podocyte cytoskeletal pro- teins in some manner, including the possibility of direct or indirect interactions between the GLEPP1 phosphatase and vimentin.

Shorter and broader foot processes occurring while slitdiaphragm width is maintained would be predicted to result in fewer foot processes, thus fewer intercellular junctions and a reduced glomerular filtration area. This observation was supported by measurement of a reduction in glomerular content of nephrin, a major podocyte intercellular junction protein. Moreover, a reduction in GFR was unmasked following uninephrectomy of $\mathrm{Ptpro}^{-/-}$mice. In addition, the $\mathrm{Ptpro}^{-/-}$mice were predisposed to hypertension, since reduction of nephron mass by removal of one or more kidneys resulted in these animals becoming hypertensive. GLEPP1 thus appears to play a role in regulating podocyte foot-process morphology, possibly by regulating cytoskeletal architecture. GLEPP1 also plays a role in preserving normal filtration area and blood pressure. Other tyrosine phosphatases have been implicated in regulating the junctions between epithelial cells $(27,28)$. It does not appear that GLEPP1 influences the morphology of the intercellular junction directly, because the width of the slit diaphragm as measured by TEM in glutaraldehyde-fixed, dehydrated thin sections was not altered in $\mathrm{Ptpro}^{-/-}$mice.

Vimentin knockout mice are markedly affected by reduction in renal mass (29). The nephrectomized vimentin $^{-/}$mouse dies within 72 hours from renal failure. This is thought to be due to changes in vascular tone, possibly as a result of alteration of the endothelin-nitric oxide balance. However, unlike the studies described in this report, the nephrectomized vimentin ${ }^{-/-}$mice had reduced blood pressure compared with wild-type mice. Further studies will be required to evaluate the possibility that the GLEPP1 receptor is also operating in part through an effect on vimentin.

We previously detected GLEPP1 phosphatase mRNA in the brain (9), and a subsequent report in the rat has localized GLEPP1 mRNA (designated RPTP-BK) to the olfactory bulb, developing neocortex, hippocampus, and thalamus (30). A truncated form of GLEPP1 (designated PTP $\phi$ ), consisting of the phosphatase domain with and without a transmembrane region, has also been identified in macrophages (31). Thus, the knock- 
out of the GLEPP1 phosphatase might possibly lead to abnormalities in these organs and systems. No obvious abnormality of development or structure of brain or hematopoietic system was seen. Furthermore, the $\mathrm{Ptpro}^{-/-}$mice are apparently capable of reproducing normally, and no obvious differences in behavior between wild-type and $\mathrm{Ptpro}^{-/-}$mice have been noted. However, no specific studies have been performed to assess brain or macrophage structure and function.

The observation that disruption of the Ptpro gene results in a predisposition to increased blood pressure provides potentially important new insight into podocyte function and raises the question of whether common forms of hypertension could also be related to podocyte dysfunction. The alternative hypothesis that the tendency to hypertension might be related to a central nervous system effect is unlikely in view of the requirement for uninephrectomy to observe the hypertension effect. Guyton showed that appropriate kidney function is necessary for long-term maintenance of systemic blood pressure (32). Clearly, the anatomic position of the podocyte at the gateway of the glomerular filter is well situated to control the glomerular pressure/filtration rate relationship. If this were to be the case, then defects of podocyte function may span a broader set of clinical conditions than was thought previously. We know that podocyte dysfunction causes increased leakiness of the glomerular filter, which manifests clinically by proteinuria and the nephrotic syndrome. We speculate that hypertension may represent the other end of the spectrum of clinical conditions caused by podocyte dysfunction where, in a sense, the glomerular filter is too tight rather than too leaky.

\section{Acknowledgments}

This work was supported by grants from the NIH (DK39255 and DK-46073). We thank Andras Nagy, Reka Nagy, and Wanda Abramow-Newerly for providing R1 ES cells, and Richard Mulligan for providing the PPNT vector. We wish to thank Chris Edwards for expert help with SEM, and Lisa Riggs and Robin Kunkel for help with TEM analysis. We also thank Sally A. Camper and Paul D. Killen for helpful advice.

1. Drenckhahn, D., and Franke, R.P. 1988. Ultrastructural organization of contractile and cytoskeletal proteins in glomerular podocytes of chicken, rat and man. Lab. Invest. 59:673-682.

2. Reiser, J., Kriz, W., Kretzler, M., and Mundel, P. 2000. The glomerular slit diaphragm is a modified adherens junction. J. Am. Soc. Nephrol. 11:1-8.

3. Kerjaschki, D., Sharkey, D.J., and Farquhar, M.G. 1984. Identification and characterization of podocalyxin, the major sialoprotein of the renal glomerular epithelial cell. J. Cell Biol. 98:1591-1596.

4. Gelberg, H., Healy, L., Whiteley, H., Miller, L.A., and Vimr, E. 1996. In vivo enzymatic removal of the $\alpha 2 \rightarrow 6$-linked sialic acid from the glomerular filtration barrier results in podocyte charge alteration and glomerular injury. Lab. Invest. 74:907-920.

5. Kreidberg, J.A., et al. 1996. Alpha 3 beta 1 integrin has a crucial role in kidney and lung organogenesis. Development. 122:3537-3547.

6. Kritz, W., Mundel, P., and Elger, M. 1994. The contractile apparatus of podocytes is arranged to counteract GBM expansion. Contrib. Nephrol. 107:1-9.

7. Seiler, M.W., Rennke, H.G., Venkatachalam, M.A., and Cotran, R.S. 1977. Pathogenesis of polycation-induced alterations ("fusion") of glomerular epithelium. Lab. Invest. 36:48-61.
8. Olson, J.L. 1992. The nephrotic syndrome. In Pathology of the kidney. R. Heptinstall, editor. Little, Brown \& Co. Boston, Massachusetts, USA/Toronto, Canada/London, United Kingdom. 779-869.

9. Thomas, P.E., et al. 1994. GLEPP1, a renal glomerular epithelial cell (podocyte) membrane protein-tyrosine phosphatase. Identification, molecular cloning, and characterization in rabbit. J. Biol. Chem. 269:19953-19962.

10. Wiggins, R.C., Wiggins, J.E., Goyal, M., Wharram, B.L., and Thomas, P.E. 1995. Molecular cloning of cDNAs encoding human GLEPP1, a membrane protein tyrosine phosphatase: characterization of the GLEPP1 protein distribution in human kidney and assignment of the GLEPP1 gene to human chromosome 12p12-p13. Genomics. 27:174-181.

11. Yang, D.H., et al. 1996. Glomerular epithelial protein 1 and podocalyxin-like protein 1 in inflammatory glomerular disease (crescentic nephritis) in rabbit and man. Lab. Invest. 74:571-584.

12. Sharif, K., Goyal, M., Kershaw, D., Kunkel, R., and Wiggins, R. 1998. Podocyte phenotypes as defined by expression and distribution of GLEPP1 in the developing glomerulus and in nephrotic glomeruli from $\mathrm{MCD}, \mathrm{CNF}$, and FSGS. A dedifferentiation hypothesis for the nephrotic syndrome. Exp. Nephrol. 6:234-244.

13. Tryggvason, K. 1999. Unraveling the mechanisms of glomerular ultrafiltration: nephrin, a key component of the slit diaphragm. J. Am. Soc. Nephrol. 10:2440-2445.

14. Shih, N.Y., et al. 1999. Congenital nephrotic syndrome in mice lacking CD2-associated protein. Science. 286:312-315.

15. Noakes, P.G., et al. 1995. The renal glomerulus of mice lacking slaminin/laminin $\beta 2$ : nephrosis despite molecular compensation by laminin $\beta 1$. Nat. Genet. 10:400-406.

16. Duhme, C., McNagny, K.M., Killen, P.D., and Kershaw, D.B. 1999. Homozygous disruption of the murine podocyte/MEP21 gene leads to disordered glomerular structure and neonatal death. J. Am. Soc. Nephrol. 10:544a. (Abstr.)

17. Kaplan, J.M., et al. 2000. Mutations in ACTN4, encoding $\alpha$-actinin-4, cause familial focal segmental glomerulosclerosis. Nat. Genet. 24:251-256.

18. Boute, N., et al. 2000. NPHS2, encoding the glomerular protein podocin, is mutated in autosomal recessive steroid-resistant nephrotic syndrome. Nat. Genet. 24:349-354.

19. Tybulewicz, V.L., Crawford, C.E., Jackson, P.K., Bronson, R.T., and Mulligan, R.C. 1991. Neonatal lethality and lymphopenia in mice with a homozygous disruption of the c-abl proto-oncogene. Cell. 65:1153-1163.

20. Nagy, A., Rossant, J., Nagy, R., Abramow-Newerly, W., and Roder, J.C. 1993. Derivation of completely cell culture-derived mice from early-passage embryonic stem cells. Proc. Natl. Acad. Sci. USA. 90:8424-8428.

21. Kendall, S.K., Samuelson, L.C., Saunders, T.L., Wood, R.I., and Camper, S.A. 1995. Targeted disruption of the pituitary glycoprotein hormone $\alpha-$ subunit produces hypogonadal and hypothyroid mice. Genes Dev. 9:2007-2019.

22. Guan, K.L., and Dixon, J.E. 1991. Eukaryotic proteins expressed in Escherichia coli: an improved thrombin cleavage and purification procedure of fusion proteins with glutathione S-transferase. Anal. Biochem. 192:262-267

23. Merritt, S.E., Killen, P.D., Phan, S.H., and Wiggins, R.C. 1990. Analysis of $\alpha 1$ (I) procollagen $\alpha 1$ (IV) collagen and $\beta$-actin mRNA in glomerulus and renal cortex of rabbits with experimental anti-glomerular basement membrane disease. Evidence for early extraglomerular collagen biosynthesis. Lab. Invest. 63:762-769.

24. Terzi, F., Beaufils, H., Laouari, D., Burtin, M., and Kleinknecht, C. 1992. Renal effect of anti-hypertensive drugs depends on sodium diet in the excision remnant kidney model. Kidney Int. 42:354-363.

25. Valgeirsdottir, S., et al. 1998. PDGF induces reorganization of vimentin filaments. J. Cell Sci. 30:1973-1980.

26. Inagaki, M., et al. 1989. Regulation of assembly-disassembly of intermediate filaments in vitro. Cell Struct. Funct. 14:279-286.

27. Tonks, N.K., and Neel, B.G. 1996. From form to function: signaling by protein tyrosine phosphatases. Cell. 87:365-368.

28. Collares-Buzato, C.B., Jepson, M.A., Simmons, N.L., and Hirst, B.H. 1998. Increased tyrosine phosphorylation causes redistribution of adherens junction and tight junction proteins and perturbs paracellular barrier function in MDCK epithelia. Eur. J. Cell Biol. 76:85-92.

29. Terzi, F., et al. 1997. Reduction in renal mass is lethal in mice lacking vimentin. Role of endothelin-nitric oxide imbalance. J. Clin. Invest. 100:1520-1528

30. Tagawa, M., et al. 1997. Identification of a receptor-type protein tyrosine phosphatase expressed in postmitotic maturing neurons: its structure and expression in the central nervous system. Biochem. J. 321:865-871.

31. Pixley, F.J., Lee, P.S., Dominguez, M.G., Einstein, D.B., and Stanley, E.R. 1995. A heteromorphic protein-tyrosine phosphatase, PTP $\phi$, is regulated by CSF-1 in macrophages. J. Biol. Chem. 270:27339-27347.

32. Guyton, A.C. 1991. Blood pressure control: special role of the kidneys and body fluids. Science. 252:1813-1816. 